\title{
Cocrystal engineering: Tuning the charge transfer excitons for highly sensitive luminescent switching materials under multiple stimuli
}

\author{
Yihan Zhang ${ }^{1 \dagger}$, Hongnan $\mathrm{Wu}^{1 \dagger}$, Yajing Sun ${ }^{1}$, Shuyu $\mathrm{Li}^{2}$, Si Liu ${ }^{1}$, Lei Zheng ${ }^{1}$, Lingjie Sun ${ }^{1,3^{*}}$, Fangxu Yang ${ }^{1}$, \\ Xiaotao Zhang ${ }^{2,4^{*}}$ and Wenping $\mathrm{Hu}^{1,3}$
}

\begin{abstract}
This paper presents a multi-stimuli responsive cocrystal system with luminescent properties that can be dynamically controlled and demonstrates the responsive mechanism of cocrystals under multiple stimuli (acid/alkali vapor, force, and heat). Detailed spectroscopic, computational, and structural studies exhibit that obvious charge transfer interactions occur in loosely mixed-stacking cocrystals. Such interactions can be weakened by acid vapor fuming due to the strong electron-withdrawing effect of acid cations and strengthened under mechanical grinding. Furthermore, the response time of the cocrystal is in the order of seconds, which is much superior to those of most previously reported stimuli-responsive cocrystals. Accordingly, a high-sensitive fluorescence switching is demonstrated under multiple stimuli, providing an effective strategy to develop smart materials.
\end{abstract}

Keywords: organic cocrystal, charge transfer, stimuli-responsive behavior, smart materials

\section{INTRODUCTION}

Organic cocrystals have gained increasing attention as an effective strategy for constructing functional materials because of their advantages, such as rational design, easy fabrication, and low cost [1-4]. They have been widely developed in organic light-emitting transistors [5], organic field-effect transistors [68], optical waveguide [9-11], pure organic room-temperature phosphorescent properties [12,13], and nonlinear optics [14]. They are connected through weak noncovalent bonds among different constituent compounds, which can be altered much easier than strong covalent bonds in inorganic crystalline materials $[15,16]$. Hence, the bulky physicochemical properties of organic cocrystals could be tuned in response to various external stimuli [17-19]. Extensive investigations have been performed on the stimuli-responsive behaviors of single-component molecules [20], but the exploration of multiple components is still in its infancy because of the unclear mechanism of cocrystallization and uncontrollable functions of final products [21]. To address this dilemma, scientists have strived to explore how to change the cocrystal properties under external stimuli, and they found that the crystal-to-crystal transition between two polymorphs could provide an intuitive demonstration of understanding the stimuli-responsive mechanism $[22,23]$. For instance, Liu et al. [24] showed that a reversible single-crystalto-single-crystal phase transition occurs in a coronene-based cocrystal, resulting in a remarkable self-healing behavior and thermo-mechanical responses. Liu et al. [25] demonstrated that a luminescent cocrystal system could undergo crystal-to-crystal phase transformation under external stimuli (solvent or heat) accompanied with luminescence switching features. Nevertheless, simultaneously obtaining two phases in one cocrystal system to unveil the structure-performance relationship remains challenging. General rules to provide deep insights into the responsive mechanism of cocrystals under various stimuli are highly desired.

Charge transfer (CT) interactions play a key role in tuning the luminescent characteristics of organic materials; such interactions can be achieved by controlling the spatial arrangement of the donor and acceptor groups in a highly twisted molecular structure but inevitably operated by painstaking synthetic procedures [26]. Cocrystal engineering provides a viable alternative solution to overcome this difficulty. Intermolecular CT interactions can serve as a type of typical driving force to promote cocrystal assembly; they are essential for exciton dissociation, photon propagation, and bandgap adjustment [27-29]. Thus, how to tailor the CT and predict the cocrystal function is an important research focus in organic cocrystals. Previous studies focused on tailoring the static properties of cocrystals by selecting the appropriate building blocks [30]. However, methods to dynamically change the cocrystal properties under external perturbations remain to be developed [31]. Organic cocrystals possess a clear stacking structure, providing a platform to study the intermolecular interactions between the constitute units [32]. Moreover, external stimuli can change the intermolecular interactions or even the stacking structure, and the variation in the degree of $\mathrm{CT}$ interactions in cocrystals can be verified and tuned by experimental methods [25]. Thus, a molecular-level structure-performance relationship based on organic CT cocrystals can be built to reveal the responsive mechanism of cocrystals and direct the development of smart cocrystals.

\footnotetext{
${ }^{1}$ Tianjin Key Laboratory of Molecular Optoelectronic Sciences, Department of Chemistry, School of Science, Tianjin University, Tianjin 300072, China

${ }^{2}$ Institute of Molecular Aggregation Science, Tianjin University, Tianjin 300072, China

${ }^{3}$ Joint School of National University of Singapore and Tianjin University, International Campus of Tianjin University, Fuzhou 350207, China

${ }^{4}$ School of Chemistry and Chemical Engineering, Qinghai Minzu University, Qinghai 810007, China

${ }^{\dagger}$ This authors contributed equally to this work.

* Corresponding authors (emails: sunlingjie@tju.edu.cn (Sun L); zhangxt@tju.edu.cn (Zhang X))
} 
In this context, we prepared the acridine-TCNB cocrystal (ATC) composed of acridine as the donor and 1,2,4,5-tetracyanobenzene (TCNB) as the acceptor. The ATC displays a mixed-stacking arrangement with strong intermolecular interactions, which contributes to the CT nature over the whole supramolecular architecture. Meanwhile, the acridine molecules in cocrystals can easily interact with the proton because of the weak alkaline nature of their pyridyl group, and the degree of CT interactions in ATC can be changed by introducing electronwithdrawing electropositive hydrogen cations. Thus, ATC can act as a stimuli-responsive cocrystal under acid/alkali vapor stimuli associated with the dynamically changing emission properties (emission color: from yellow-green to green to orange; photoluminescence quantum yield (PLQY, $\Phi_{\mathrm{f}}$ ): from high to low and then recovers to high). ATC is sensitive to other stimuli, such as grinding and heat, and its luminescent properties can be reversibly regulated. The responsive mechanism of ATC under multiple stimuli is elaborately demonstrated, which is rarely reported in cocrystals. The responsive time of the cocrystal is in the order of seconds, which is much superior to those of most previously reported stimuli-responsive cocrystals. Therefore, we demonstrate a reversible luminescent switching cocrystal system that can be dynamically controlled under multiple stimuli through the rational design of each component, providing the desired strategy to develop next-generation smart materials [33].

\section{EXPERIMENTAL SECTION}

\section{Materials}

Acridine $\left(\mathrm{C}_{13} \mathrm{H}_{9} \mathrm{~N}, 98 \%\right)$ and TCNB $\left(\mathrm{C}_{10} \mathrm{H}_{2} \mathrm{~N}_{4}, 98 \%\right)$ were purchased from Aladdin Industrial Corporation and TCI Shanghai Company, respectively. Acetone $\left(\mathrm{C}_{3} \mathrm{H}_{6} \mathrm{O}, \mathrm{AR}\right)$ was purchased from Damao Chemical Reagent Factory. Hydrochloric acid $(\mathrm{HCl}, \mathrm{AR})$ and ammonia solution $\left(\mathrm{NH}_{3}, \mathrm{GR}\right)$ were purchased from Tianjin Fengchuan Chemical Reagent Co., Ltd. and Tianjin Kemiou Chemical Reagent Co., Ltd., respectively.

\section{Crystal growth and characterization}

In a typical experiment, TCNB and acridine were mixed and dissolved in acetone with a molar ratio of 1:1, and ATC was obtained by solvent evaporation. The crystal structure was detected using X-ray diffraction (XRD, X-Pert, PANalytic, Netherlands) with $\mathrm{Cu} \mathrm{Ka}$ radiation $(40 \mathrm{kV}, 30 \mathrm{~mA})$. Powder XRD (PXRD) patterns were recorded on a Rigaku SmartLab 9 $\mathrm{KW}$ with $\mathrm{Cu} \mathrm{Ka}$ radiation $(\lambda=1.542 \AA)$ operating at $45 \mathrm{kV}$ and $200 \mathrm{~mA}$. Solid-state UV-vis analysis was performed on a Shimadzu UV-3600 Plus spectrophotometer at room temperature. Fourier transform infrared spectra (FTIR) were obtained on a Bruker Vertex 70 FTIR spectrometer. Thermogravimetric analysis (TGA) was carried out on a Mettler Toledo TGA 2 thermal analysis system from room temperature to $400^{\circ} \mathrm{C}$ under a nitrogen atmosphere $(20 \mathrm{sccm})$. Differential scanning calorimetry (DSC) was performed using a DSC 214 polyma with a heating rate of $10 \mathrm{~K} \mathrm{~min}^{-1}$. In the acid/alkali vapor stimuliresponsive experiment, the mass percentages of hydrochloric acid and ammonia were $37 \%$ and $27 \%$, respectively, and the corresponding saturated vapor concentration was obtained at $25^{\circ} \mathrm{C}$. Each cocrystal film $\left(1 \times 1 \mathrm{~cm}^{2}\right)$ contained an average of $0.4 \mathrm{mg}$ sample. The contact area with the material was $1 \mathrm{~cm}^{2}$. The reaction was very rapid under these conditions.

\section{Theoretical calculations}

Intermolecular potentials were calculated using the UNI force field by Mercury software (copyright CCDC) [34,35]. The surface electrostatic potential (ESP) was calculated using MOPAC by Mercury software [36,37]. The calculated Bravais Friedel Donnay Harker (BFDH) morphology was shown by Mercury software according to the crystal structure. Moreover, the energy levels were obtained at the B3lyp/6-31G(d) level of theory by Gaussian 09 package. The molecular orbital surfaces were visualized with GaussianView.

\section{Optical characterizations}

The steady photoluminescence (PL) spectra were recorded with an Edinburgh instrument (FLS1000). The absolute $\Phi_{\mathrm{f}}$ was measured using an integrating sphere on an Edinburgh FLS1000 spectrometer. Luminescence decays in the nanosecond were measured with the same apparatus and class 3B laser product (EPL-375) as excitation source in multichannel scaling mode. Fluorescence micrographs were obtained using a Leica DM2700M optical microscope $(340-380 \mathrm{~nm})$ with a plug-in fluorescence unit.

\section{RESULTS AND DISCUSSION}

\section{Cocrystal preparation and crystal structure analysis}

The ATC shows a yellow color different from those of their constituent components, wherein TCNB and acridine crystals exhibit white and cream colors, respectively (Fig. 1a, above). The PXRD patterns in Fig. 1b display that ATC has different peaks from the co-former, indicating the successful cocrystallization instead of the simple sample mixing between different compounds [38]. Analysis of single crystal XRD indicates that ATC is triclinic and belongs to the space group $P-1$. The cell parameters of $a=7.43 \AA, b=7.87 \AA, c=8.07 \AA, \alpha=73.87^{\circ}, \beta=$ $84.60^{\circ}, \gamma=85.85^{\circ}$ are obtained (Table S1) [39]. The acridine molecule in ATC is disordered, and the $\mathrm{N}$ and $\mathrm{CH}$ groups at the molecular center are equivalent. Consequently, centers $\mathrm{C}$ and $\mathrm{N}$ of the acridine molecule each have $50 \%$ occupancy. As shown in Fig. 1c, d, ATC shows a mixed stack packing structure, wherein their donor and acceptor molecules are stacked face to face (Fig. 1c), which is different from that of constitute units (Fig. S1). The intermolecular D-A distance of ATC along the $a$ axis is $3.377 \AA$ (short contact), indicating the strong D-A interactions in the cocrystal [40].

Meanwhile, the molecules of ATC become more compact owing to intermolecular -N...HC- interaction in the cocrystal (Fig. 1d), and the distances of $-\mathrm{N}$...HC- between adjacent column D-A molecules are 2.673 and $2.713 \AA$, and that of $\mathrm{A}-\mathrm{A}$ molecules is $2.604 \AA$. Morphology prediction was performed based on the BFDH model by using Mercury software. ATCs grow into cube block crystals, which is in accordance with cocrystal practical growth (Fig. 1e and Fig. S2). The strongest intermolecular potential $\left(-58.1 \mathrm{~kJ} \mathrm{~mol}^{-1}\right)$ in ATC is the donoracceptor interaction along the $a$-axis, which is much stronger than those of constitute units (Fig. S3c, d), indicating the primary driving force for the self-assembly of ATC (Fig. S3b). The thermal stabilities of the cocrystals and the single components were compared and analyzed. The smooth curves of TGA indicate the absence of solvent molecules in ATC (Fig. S4b). The DSC diagram (Fig. 1f) demonstrates that the melting point of the cocrystals is different from those of their single-component 

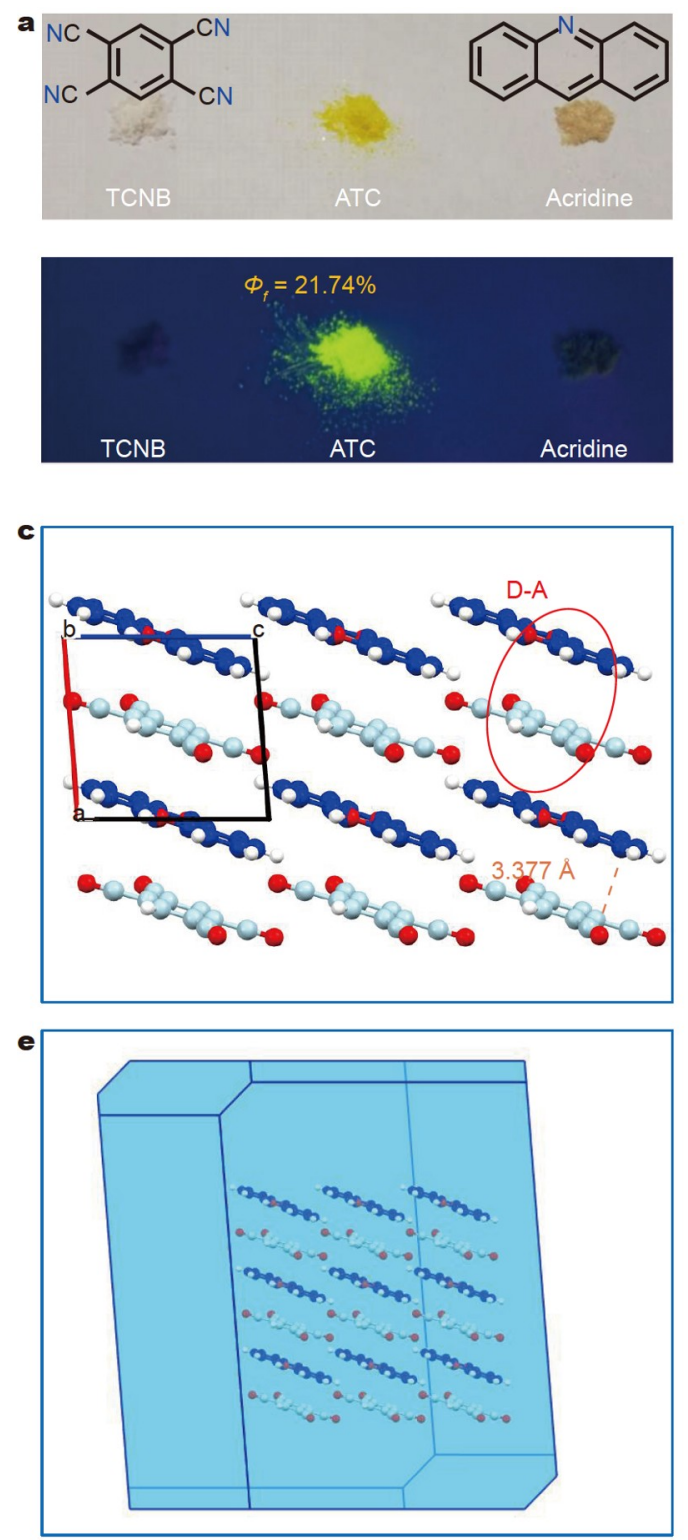
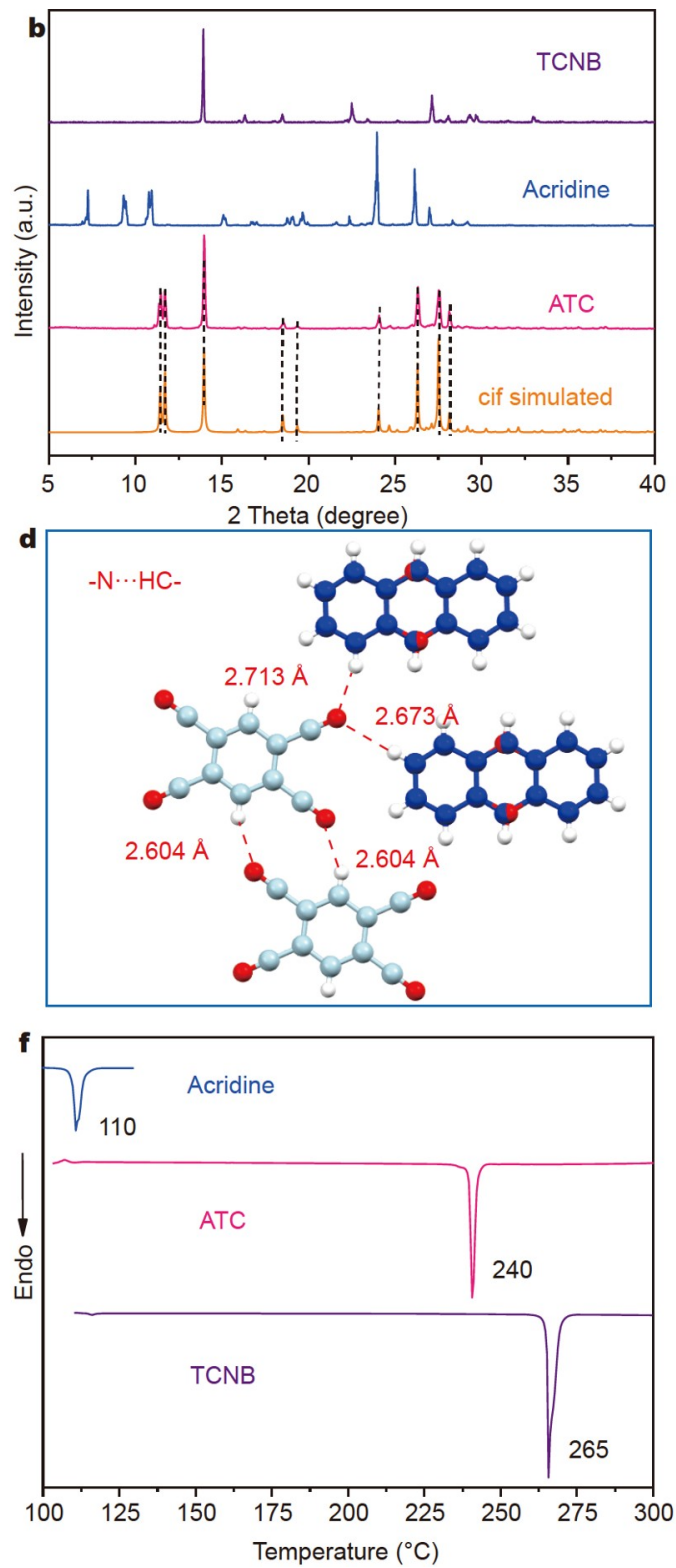

Figure 1 Optical images of TCNB, ATC, and acridine powder under (a) visible light (above) and UV lamp ( $365 \mathrm{~nm})$ (below) with the molecular structures. (b) PXRD spectra of acridine, ATC cocrystal, and TCNB. (c, d) Packing structures of ATC. (e) Simulated growth morphology of ATC. (f) DSC spectra of Acridine, ATC, and TCNB crystals.

crystals and confirms the stable structure of the cocrystals, suggesting the formation of cocrystals with a new crystal lattice.

\section{CT nature in ATC}

The spectra were studied to determine their photophysical properties. The FTIR spectrum of ATC is the combination of the single-component donor and acceptor, and the peaks of the cocrystals are very sharp, indicating that the cocrystals have high crystal quality (Fig. S4a). In addition, the $\mathrm{C} \equiv \mathrm{N}$ and the $\mathrm{C}-\mathrm{H}$ bonds on the benzene rings of TCNB are highly sensitive to the changes in the surrounding chemical environment, which is strong evidence of the existence of CT between the molecules. For example, in ATC, the TCNB band at $3112 \mathrm{~cm}^{-1}(\mathrm{C}-\mathrm{H}$ stretch) is shifted to $3107 \mathrm{~cm}^{-1}$, that at $3047 \mathrm{~cm}^{-1}$ (C-H stretch) is shifted to $3042 \mathrm{~cm}^{-1}$, and that at $2245 \mathrm{~cm}^{-1}$ ( $\mathrm{C} \equiv \mathrm{N}$ stretch) is shifted to $2243 \mathrm{~cm}^{-1}$. Fig. $2 \mathrm{~b}$ exhibits the UV-vis absorption spectra. ATC shows a new broad absorption peak with a prominent redshift at $440 \mathrm{~nm}$ compared with the individual molecular crystals. This result can be attributed to the strong CT interactions in the cocrystal system [41]. ATC has strong yellowgreen fluorescence under UV light $(365 \mathrm{~nm})$, whereas the singlecomponent TCNB and acridine molecules have no significant fluorescence (Fig. 1a, below). The fluorescence spectra were recorded to study the luminescent properties of the cocrystals (Fig. 2c). Compared with single-component molecules, the emission peak of ATC has an apparent redshift similar to the situation of the UV-vis absorption spectra. From the energy level diagram and molecular orbital diagram obtained by density functional theory calculations (Fig. 2a), the highest occupied molecular orbitals of the cocrystals are mainly concentrated on the donor molecules with an energy value of $-6.06 \mathrm{eV}$, whereas the lowest unoccupied molecular orbitals of the cocrystals are 
a
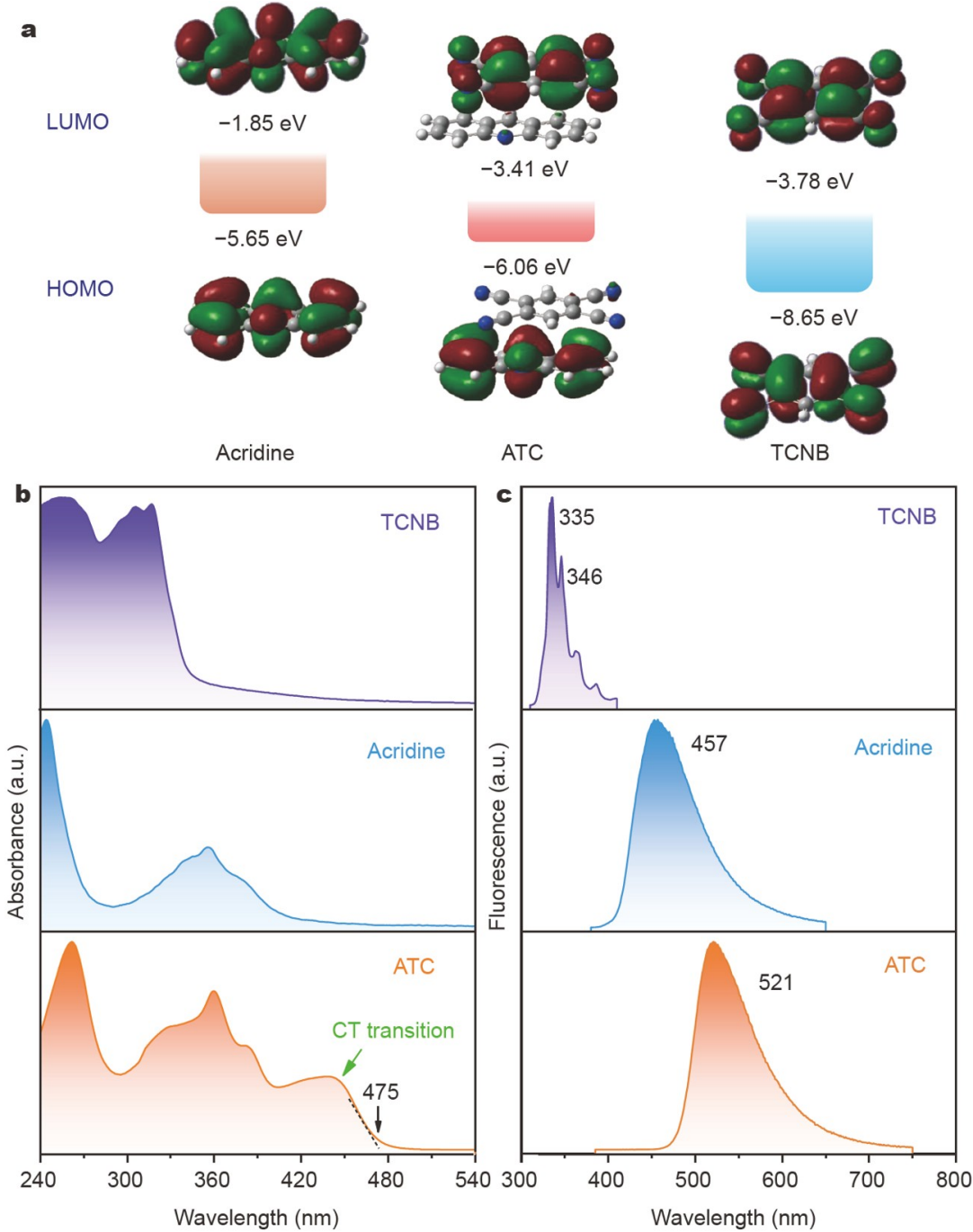

Figure 2 (a) Calculated energy level diagrams and molecular orbital diagrams of TCNB, acridine, and ATC. Solid-state (b) UV-vis absorption and (c) fluorescence spectra of TCNB, Acridine, and ATC.

concentrated on the acceptor molecules with an energy value of $-3.41 \mathrm{eV}$. Thus, the calculated energy gap of the cocrystal $(2.65 \mathrm{eV})$ is smaller than those of the single components $(3.8 \mathrm{eV}$ for acridine molecules and $4.87 \mathrm{eV}$ for TCNB molecules, respectively). Hence, ATC reveals a redshifted and broad profile in the absorption spectrum compared with the single components [42]. Moreover, the emission spectrum of ATC displays a new structureless wide peak (full width at half-maximum (FWHM): $76 \mathrm{~nm}$ ) with a large Stokes shift $\left(3533 \mathrm{~cm}^{-1}\right)$ (Fig. S5) at $521 \mathrm{~nm}$, suggesting that evident CT will occur between the donor and acceptor molecules [26].

The chromaticity coordinates (CIE) of ATC calculated from its fluorescence spectrum are $(0.33,0.60)$ (as shown in Fig. S6), and its color agrees with the fluorescence image under UV light. A double exponential decay of ATC with an average lifetime of $28.22 \mathrm{~ns}$ was obtained by time-resolved emission spectroscopic measurements (Fig. S7b). The absolute value of the $\Phi_{\mathrm{PL}}$ is $21.74 \%$, which is much higher than that of the single-component solid (Table S2).

Acid/alkali vapor stimuli

Acridine contains an $\mathrm{N}$ atom on the pyridine ring with a weakly alkaline, which can fairly easily to interact with protons to form pyridinium salts $[43,44]$. Therefore, acridine single crystal might respond to $\mathrm{HCl}$. Analysis of the response of acridine monomer before and after $\mathrm{HCl}$ stimulation (Fig. S8a) reveals that its color will change from milky white to light yellow. The fluorescence emission of acridine is also enhanced (Fig. S8b). The present work aims to prepare acridine-based CT cocrystals and analyze the changes in the degree of $\mathrm{CT}$ in response to multiple stimuli. Thus, the stimuli-responsive luminescent behaviors of the solidstate ATC were explored during protonation-deprotonation. As shown in Fig. 3a, b, and Fig. S9a, the fluorescence color of the treated ATC samples (called ATCH) changes from yellow-green to green, with the luminescence intensity gradually decreasing with time, and remains unchanged until $60 \mathrm{~s}$ after the ATC samples are exposed to $\mathrm{HCl}$ vapor. The responsive time of ATC is faster than those of most previously reported cocrystals $[25,45]$. Conversely, the alkalized ATCHN samples, referring to the ATCH samples exposed to alkaline $\mathrm{NH}_{3}$ vapor, show orange emission with an increasing luminescence intensity and remain unchanged until $30 \mathrm{~s}$ after being fumed with the $\mathrm{NH}_{3}$ vapor (Fig. 3a, c, and Fig. S9b). Moreover, ATCH shows a shorter lifetime than the initial ATC samples, with a value of $5.60 \mathrm{~ns}$, 


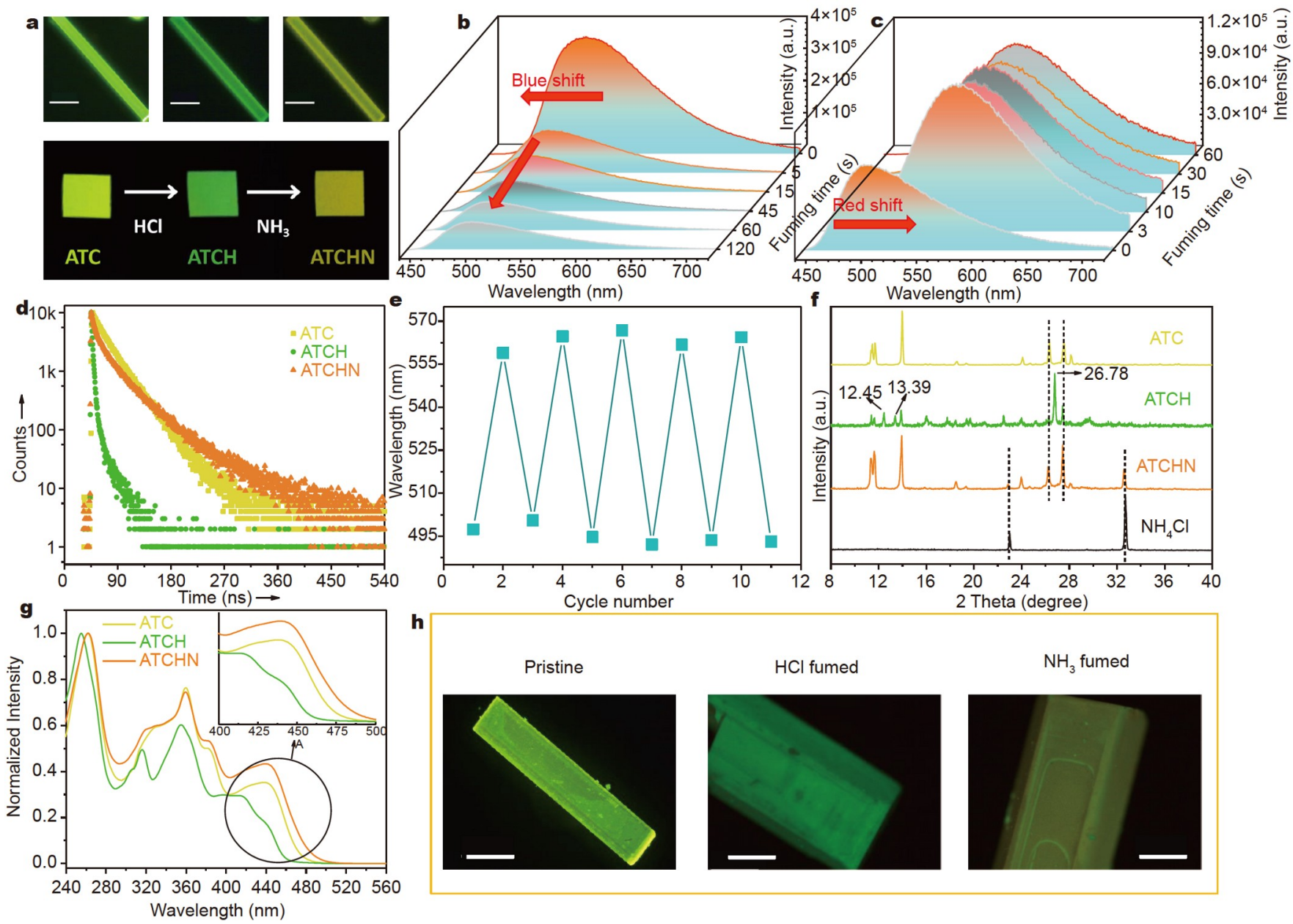

Figure 3 (a) Fluorescent images of ATC, ATCH, and ATCHN. Above: crystal (scale bar: $50 \mu \mathrm{m})$; below: ground powder film $\left(1 \times 1 \mathrm{~cm}^{2}\right)$. Time-dependent photoluminescence spectra of ATC exposed to (b) $\mathrm{HCl}$ vapor and (c) $\mathrm{NH}_{3}$ vapor. (d) Time-resolved fluorescence measurements of ATC, ATCH, and ATCHN. (Monitored at $375 \mathrm{~nm}$ ). (e) Cycles of the fluorescent change and recovery by exposing ATC to $\mathrm{NH}_{3}$ and $\mathrm{HCl}$ vapors, respectively. (f) PXRD and (g) UV-vis absorption spectra of ATC, ATCH, and ATCHN. (h) Images of ATC surface, ATCH, and ATCHN under a fluorescence microscope. Scale bar: $500 \mu \mathrm{m}$.

and the PLQY is down to 5.43\%. However, ATCHN exhibits a longer life with a value of $42.20 \mathrm{~ns}$ and a moderate PLQY value of $11.80 \%$ (Fig. $3 \mathrm{~d}$ and Fig. S7, and Table S2). Furthermore, the reversible radiative oscillation can be readily repeated at least five times without any fatigue (Fig. 3e and Fig. S10). As shown in Fig. $3 \mathrm{~h}, \mathrm{ATCH}$ and ATCHN both display a rough and turbid surface relative to the smooth surface of ATC, which further confirms that the acid/alkali vapor stimuli occur on the crystal surface (Fig. S11).

The PXRD patterns of ATC, ATCH, and ATCHN were investigated to understand this phenomenon. The PXRD pattern of ATCH shows some new, sharp, and well-defined peaks at $12.45^{\circ}, 13.39^{\circ}$, and $26.78^{\circ}$ (Fig. 3f), and some peaks of ATC disappear. This result indicates that the introduction of $\mathrm{HCl}$ changes the crystal structure of ATC during fuming and that ATCH is a new material with high crystallinity. Moreover, the PXRD peaks of ATCHN almost correspond to the pattern of initial ATC, except for the new peaks at $22.86^{\circ}$ and $32.26^{\circ}$, which belong to the pattern of $\alpha$-ammonium chloride. This result indicates that the ATCHN samples are a mixture of initial ATC and ammonium chloride salts. The UV-vis absorption spectra of the ATC cocrystal were obtained (Fig. 3g). The absorption region of $\mathrm{ATCH}$ around $440 \mathrm{~nm}$, which is attributed to the CT interactions, has a blue shift relative to the initial ATC. Thus, the bandgap of ATCH becomes wider than that of ATC, and the donor-acceptor interactions weaken in $\mathrm{ATCH}$, which arises from the electron cloud rearrangement due to the introduction of electron-withdrawing $\mathrm{H}^{+}$during fuming [46]. In addition, the absorption spectrum of ATCHN is redshifted to long wavelengths compared with that of ATC. Thus, the bandgap of ATCHN becomes narrower than that of ATC, and the donoracceptor interactions strengthen in ATCHN, which may arise from the crystalline transformation in the final state. The changes in the FWHM and the fluorescence emission spectrum of ATC in direct contact with $\mathrm{NH}_{3}$ vapor also indicate that external stimuli can change the fluorescence properties (Fig. S12) [47].

\section{Other stimuli}

$\mathrm{HCl}$ molecules can be introduced into ATC in the acid/alkali vapor stimuli process, suggesting that ATC displays a loose molecular packing. Thus, whether the molecular structure or the intermolecular interactions can be altered under other stimuli, such as force or heat, was explored further. As expected, the 
fluorescence color of the ground ATC changes from yellowgreen to orange, and a redshift (from 521 to $560 \mathrm{~nm}$ ) is observed relative to the initial ATC for $60 \mathrm{~s}$ (Fig. 4b). The ground ATC shows a longer lifetime than the initial ATC samples with a value of $40.40 \mathrm{~ns}$ (Fig. S13). The PXRD pattern of ground ATC exhibits a shift toward a low angle with relatively weak and broad features, indicating that those intermolecular interactions are increased by anisotropic grinding, leading to a much tighter structure than ATC (Fig. 4c and Fig. S14) [48]. Meanwhile, the absorption spectrum of the ground ATC displays a slight redshift relative to the initial ATC, which indicates the structure rearrangement after grinding. This phenomenon is responsible for the change in the luminescent properties (Fig. S15). Furthermore, grinding ATC powders and then heating to $130^{\circ} \mathrm{C}$ restore them to the initial state (Fig. 4a), and the reversible process can be readily repeated at least five times without any fatigue (Fig. 4d and Fig. S16). Thus, a multi-stimuli responsive programmable fluorescence memory system can be realized in ATC (Fig. 4e). When the ATC is fumed by acidic vapor, the yellow-green emission can blue-shift into green, whereas the yellow-green emission of ATC can redshift into orange emission by grinding. The fumed ATCH can also reversibly turn into orange emission after being treated by alkalic vapor.

\section{Multi-stimuli-responsive mechanism of ATC}

A clear overall relationship between the stimuli type and the luminescent properties must be established to reveal the origin of the multi-stimuli-responsive behavior of ATC. The surface ESP can also provide a clear understanding of the flow direction of electrons in the cocrystals. However, the ESP of ATC cannot be calculated because of its disordered crystal structure. The calculated ESP of single components can provide some clues. As shown in Fig. 5a, the red regions with the electron-rich feature are located on the center of the acridine molecule, whereas the blue regions with the electron-deficient feature are concentrated on the benzene ring of the TCNB molecule. Therefore, free electrons will move from acridine to TCNB in the mixedstacking structure after cocrystal formation (Fig. 5b), wherein $\mathbf{a}$
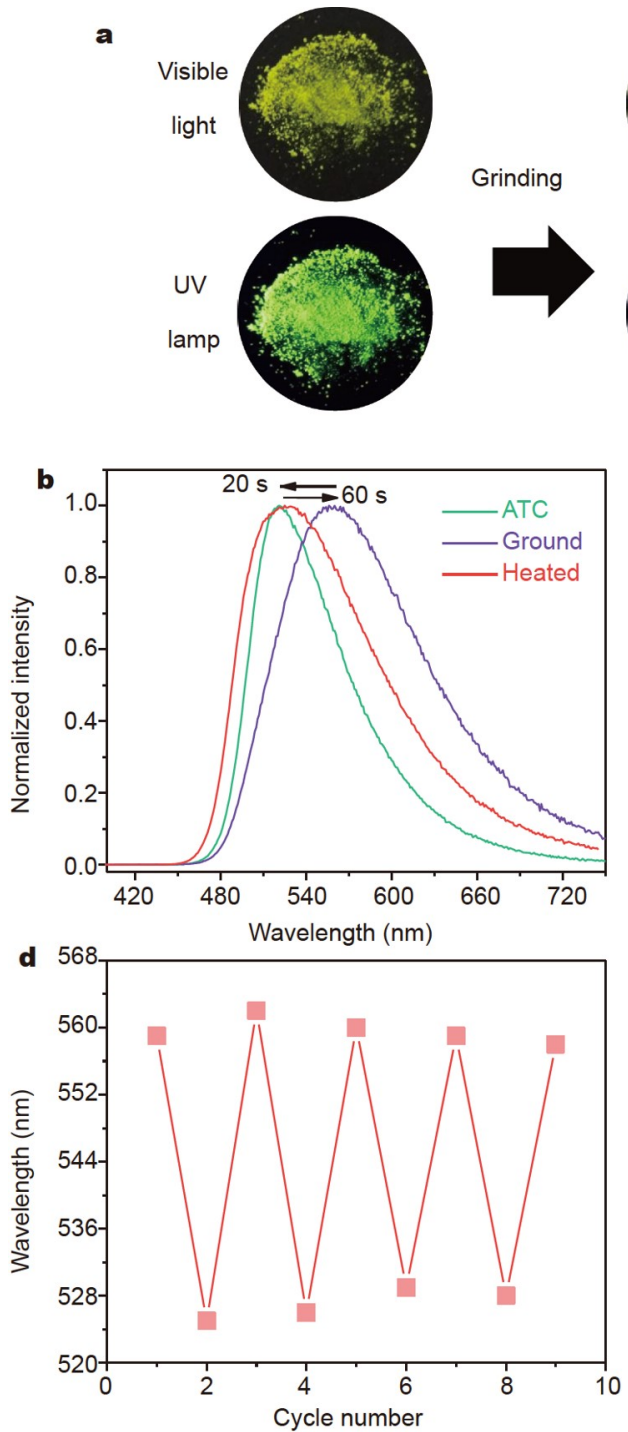
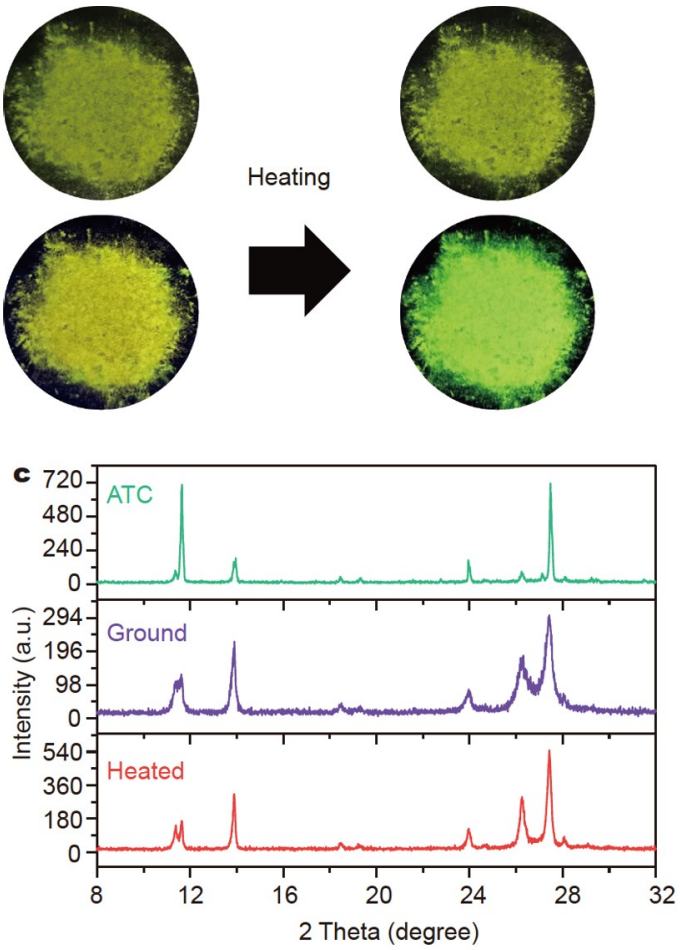

e

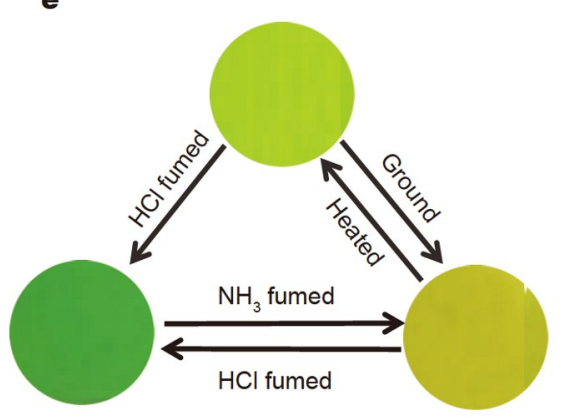

Figure 4 (a) Fluorescent images of ATC, ground-ATC, and heated-ATC. Above: visible light; below: UV lamp (365 nm). (b) Fluorescence spectra and (c) PXRD of ATC, ground-ATC, and heated-ATC. (d) Cycles of the fluorescent change and recovery by grinding and heating ATC, respectively. (e) Diagram demonstration of the fluorescence system cycle of ATC. 
a
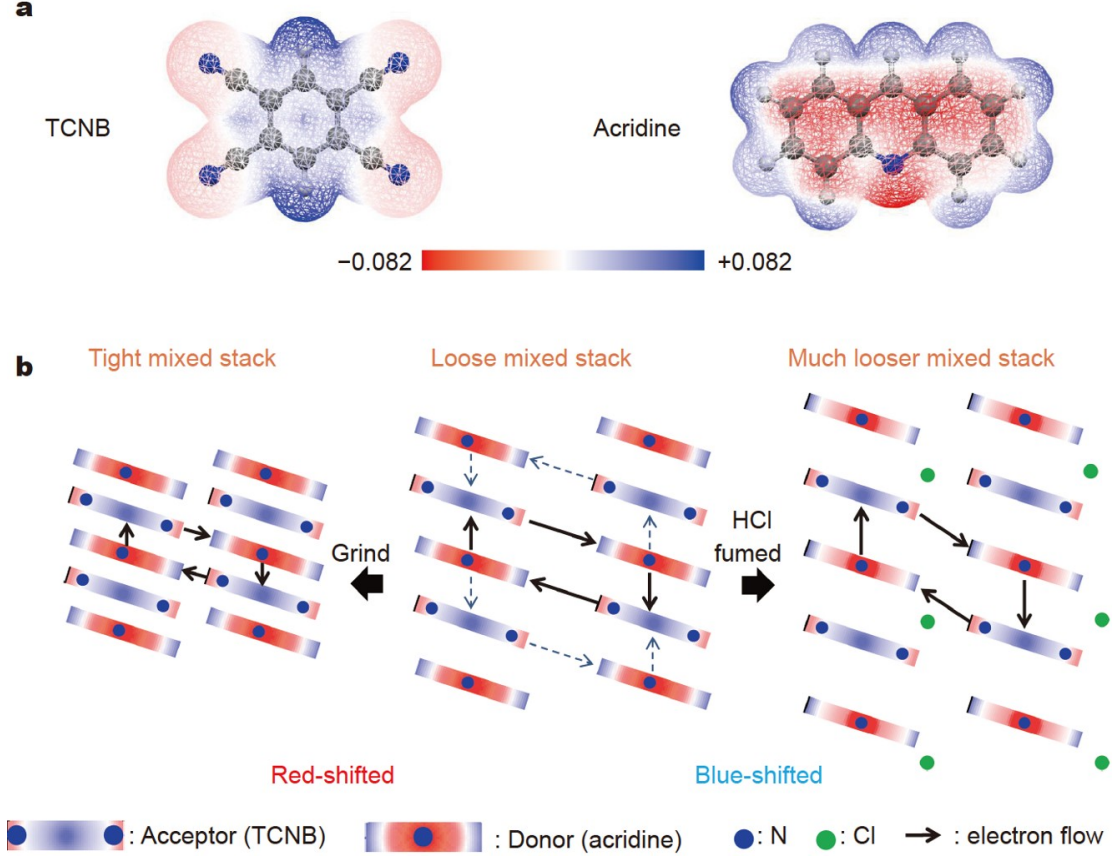

Figure 5 (a) View the structure's van der Waals surface of TCNB and acridine, colored-coded by the ESP. Unit: eV. (b) Model schematic of molecular stacking mode in ATC under different stimuli.

the intermolecular interaction in the same column will drive the cocrystal assembly. This phenomenon is in accordance with the result of the calculated strongest intermolecular potentials in the cocrystals (Fig. S3b). Upon acid vapor fuming, the electropositive hydrogen atom of $\mathrm{HCl}$ connects with the electronegative $\mathrm{N}$ atom of acridine by a strong hydrogen bond to form a pyridinium salt. The newly formed pyridinium salt of ATCH exhibits a much looser structure than ATC because of the steric hindrance of chlorine anion, whereas the electron-withdrawing effect of $\mathrm{H}^{+}$in $\mathrm{ATCH}$ will reduce the electron cloud density of the donor molecules and the degree of CT between the donor and acceptor molecules. This collaborative effect is responsible for the blue shift of the luminescence spectrum of ATC upon acid vapor fuming. Meanwhile, the mechanical grinding can induce the structure rearrangement [49], and make the bandgap narrower and intermolecular interactions stronger than those of original ATC, which accounts for the redshift of the luminescence spectrum of ATC under an external force. Thus, the CT interactions of ATC can be tuned, and the luminescent properties of ATC are notably influenced to respond to various stimuli. Similarly, the high-luminescence organoboron complex also has a multi-stimuli-responsive behavior [50,51]. Therefore, cocrystallization lays the foundation for the design of multistimuli-responsive materials.

\section{CONCLUSION}

ACT with a 1:1 stoichiometric ratio was obtained by molecular self-assembly. The cocrystal exhibits a mixed-stacking mode with obvious CT features, which is responsible for the unique PL properties relative to the individual units. Moreover, ATC displays a sensitive response to external stimuli (acid/alkali vapor, grinding, and heat), and the intermolecular CT interactions can be controllably tuned, leading to dynamical change of the cocrystal properties (i.e., the emission color, lifetime, and PLQY) under external perturbations. This result indicates that organic cocrystals with the CT nature are a type of promising candidate for building a molecular-level structure-performance relationship to reveal the responsive mechanism of cocrystals under external stimuli and their great potential applications in fluorescence sensors.

Received 11 September 2021; accepted 3 November 2021; published online 7 December 2021

1 Sun L, Wang Y, Yang F, et al. Cocrystal engineering: A collaborative strategy toward functional materials. Adv Mater, 2019, 31: 1902328

2 Huang Y, Wang Z, Chen Z, et al. Organic cocrystals: Beyond electrical conductivities and field-effect transistors (FETs). Angew Chem Int Ed, 2019, 58: 9696-9711

3 Park SK, Kim JH, Park SY. Organic 2D optoelectronic crystals: charge transport, emerging functions, and their design perspective. Adv Mater, 2018, 30: 1704759

4 Wuest JD. Co-crystals give light a tune-up. Nat Chem, 2012, 4: 74-75

5 Park SK, Kim JH, Ohto T, et al. Highly luminescent 2D-type slab crystals based on a molecular charge-transfer complex as promising organic light-emitting transistor materials. Adv Mater, 2017, 29: 1701346

6 Sakai M, Sakuma H, Ito $\mathrm{Y}$, et al. Ambipolar field-effect transistor characteristics of (BEDT-TTF)(TCNQ) crystals and metal-like conduction induced by a gate electric field. Phys Rev B, 2007, 76: 045111

7 Zhang J, Geng H, Virk TS, et al. Sulfur-bridged annulene-TCNQ Cocrystal: A self-assembled "molecular level heterojunction" with air stable ambipolar charge transport behavior. Adv Mater, 2012, 24: 26032607

8 Wu HD, Wang FX, Xiao Y, et al. Preparation and ambipolar transistor characteristics of co-crystal microrods of dibenzotetrathiafulvalene and tetracyanoquinodimethane. J Mater Chem C, 2013, 1: 2286-2289

9 Zhu W, Zheng R, Fu X, et al. Revealing the charge-transfer interactions in self-assembled organic cocrystals: Two-dimensional photonic applications. Angew Chem Int Ed, 2015, 54: 6785-6789

10 Lei YL, Liao LS, Lee ST. Selective growth of dual-color-emitting heterogeneous microdumbbells composed of organic charge-transfer 
complexes. J Am Chem Soc, 2013, 135: 3744-3747

11 Lu B, Fang X, Yan D. Luminescent polymorphic co-crystals: A promising way to the diversity of molecular assembly, fluorescence polarization, and optical waveguide. ACS Appl Mater Interfaces, 2020, 12: 31940-31951

12 d'Agostino S, Grepioni F, Braga D, et al. Tipping the balance with the aid of stoichiometry: Room temperature phosphorescence versus fluorescence in organic cocrystals. Cryst Growth Des, 2015, 15: 20392045

13 Bolton O, Lee K, Kim HJ, et al. Activating efficient phosphorescence from purely organic materials by crystal design. Nat Chem, 2011, 3: 205-210

14 Sun L, Zhu W, Wang W, et al. Intermolecular charge-transfer interactions facilitate two-photon absorption in styrylpyridine-tetracyanobenzene cocrystals. Angew Chem Int Ed, 2017, 56: 7831-7835

15 Braga D, Maini L, Grepioni F. Mechanochemical preparation of cocrystals. Chem Soc Rev, 2013, 42: 7638-7648

16 Ji W, Xue B, Bera S, et al. Tunable mechanical and optoelectronic properties of organic cocrystals by unexpected stacking transformation from H- to J- and X-aggregation. ACS Nano, 2020, 14: 10704-10715

17 Mutai T, Satou H, Araki K. Reproducible on-off switching of solid-state luminescence by controlling molecular packing through heat-mode interconversion. Nat Mater, 2005, 4: 685-687

18 Bai L, Bose P, Gao Q, et al. Halogen-assisted piezochromic supramolecular assemblies for versatile haptic memory. J Am Chem Soc, 2017, 139: 436-441

19 Li S, Yan D. Tuning light-driven motion and bending in macroscaleflexible molecular crystals based on a cocrystal approach. ACS Appl Mater Interfaces, 2018, 10: 22703-22710

20 Wang Y, Yang J, Gong Y, et al. Host-guest materials with room temperature phosphorescence: Tunable emission color and thermal printing patterns. SmartMat, 2020, 1: e1006

21 Sun L, Yang F, Zhang X, et al. Stimuli-responsive behaviors of organic charge transfer cocrystals: Recent advances and perspectives. Mater Chem Front, 2020, 4: 715-728

22 Bolton O, Matzger AJ. Improved stability and smart-material functionality realized in an energetic cocrystal. Angew Chem Int Ed, 2011, 50: $8960-8963$

23 Morimoto $\mathrm{M}$, Irie M. A diarylethene cocrystal that converts light into mechanical work. J Am Chem Soc, 2010, 132: 14172-14178

24 Liu G, Liu J, Ye X, et al. Self-healing behavior in a thermo-mechanically responsive cocrystal during a reversible phase transition. Angew Chem Int Ed, 2017, 56: 198-202

25 Liu Y, Li A, Xu S, et al. Reversible luminescent switching in an organic cocrystal: Multi-stimuli-induced crystal-to-crystal phase transformation. Angew Chem Int Ed, 2020, 59: 15098-15103

26 Sun L, Hua W, Liu Y, et al. Thermally activated delayed fluorescence in an organic cocrystal: Narrowing the singlet-triplet energy gap via charge transfer. Angew Chem Int Ed, 2019, 58: 11311-11316

27 Wang Z, Yu F, Xie J, et al. Insights into the control of optoelectronic properties in mixed-stacking charge-transfer complexes. Chem Eur J, 2020, 26: 3578-3585

28 Oh S, Park SK, Jhun BH, et al. Unraveling the origin of high-efficiency photoluminescence in mixed-stack isostructural crystals of organic charge-transfer complex: Fine-tuning of isometric donor-acceptor pairs. J Phys Chem C, 2020, 124: 20377-20387

29 Hu P, Du K, Wei F, et al. Crystal growth, HOMO-LUMO engineering, and charge transfer degree in perylene- $\mathrm{F}_{x} \mathrm{TCNQ}(x=1,2,4)$ organic charge transfer binary compounds. Cryst Growth Des, 2016, 16: 30193027

30 Jin J, Wu S, Ma Y, et al. Nucleation control-triggering cocrystal polymorphism of charge-transfer complexes differing in physical and electronic properties. ACS Appl Mater Interfaces, 2020, 12: 1971819726

31 Ye X, Liu Y, Guo Q, et al. 1D versus $2 \mathrm{D}$ cocrystals growth via microspacing in-air sublimation. Nat Commun, 2019, 10: 761

32 Liu Y, Zeng Q, Zou B, et al. Piezochromic luminescence of donoracceptor cocrystals: Distinct responses to anisotropic grinding and isotropic compression. Angew Chem Int Ed, 2018, 57: 15670-15674
$33 \mathrm{Hu} \mathrm{W}$, Zhang H, Salaita K, et al. SmartMat: Smart materials to smart world. SmartMat, 2020, 1: smm2.1014

34 Gavezzotti A, Filippini G. Geometry of the intermolecular X-H...Y (X, Y $=\mathrm{N}, \mathrm{O})$ hydrogen bond and the calibration of empirical hydrogenbond potentials. J Phys Chem, 1994, 98: 4831-4837

35 Dunitz JD. Are crystal structures predictable? Chem Commun, 2003, : 545-548

36 Freire RO, Rocha GB, Simas AM. Modeling rare earth complexes: Sparkle/AM1 parameters for thulium(III). Chem Phys Lett, 2005, 411: 61-65

37 Rocha GB, Freire RO, Simas AM, et al. RM1: A reparameterization of AM1 for H, C, N, O, P, S, F, Cl, Br, and I. J Comput Chem, 2006, 27: 1101-1111

38 Huang Y, Gong Q, Ge J, et al. Green grinding-coassembly engineering toward intrinsically luminescent tetracene in cocrystals. ACS Nano, 2020, 14: 15962-15972

39 Marsh RE. 1:1 Molecular complex of acridine with 1,2,4,5-benzenetetracarbonitrile: refinement in space group $P \overline{1}$. Acta Crystlogr C Cryst Struct Commun, 1990, 46: 1356-1357

40 Wu HD, Wang FX, Zhang M, et al. Investigation of transport properties of coronene-TCNQ cocrystal microrods with coronene microrods and TCNQ microsheets. Nanoscale, 2015, 7: 12839-12842

41 Sun L, Zhu W, Yang F, et al. Molecular cocrystals: Design, chargetransfer and optoelectronic functionality. Phys Chem Chem Phys, 2018, 20: 6009-6023

42 Sun Y, Lei Y, Liao L, et al. Competition between arene-perfluoroarene and charge-transfer interactions in organic light-harvesting systems. Angew Chem Int Ed, 2017, 56: 10352-10356

43 Zhang J, Chen J, Xu B, et al. Remarkable fluorescence change based on the protonation-deprotonation control in organic crystals. Chem Commun, 2013, 49: 3878-3880

44 Ma S, Zhang J, Liu Y, et al. Direct observation of the symmetrical and asymmetrical protonation states in molecular crystals. J Phys Chem Lett, 2017, 8: 3068-3072

45 Li S, Yan D. A reversible acid-base fluorescent switch based on molecular cocrystal of 4-[2-4-quinolinyl)vinyl]phenol and tetrafluoroterephthalic acid. Sci China Chem, 2018, 61: 215-221

46 Li ZZ, Wang XD, Liao LS. Luminescence-/morphology-modulation of organic microcrystals by a protonation process. J Mater Chem C, 2017, 5: $6661-6666$

47 Xiong J, Wang K, Yao Z, et al. Multi-stimuli-responsive fluorescence switching from a pyridine-functionalized tetraphenylethene AIEgen. ACS Appl Mater Interfaces, 2018, 10: 5819-5827

48 Yan D, Yang H, Meng Q, et al. Two-component molecular materials of 2,5-diphenyloxazole exhibiting tunable ultraviolet/blue polarized emission, pump-enhanced luminescence, and mechanochromic response. Adv Funct Mater, 2014, 24: 587-594

49 Wang Z, Yu F, Chen W, et al. Rational control of charge transfer excitons toward high-contrast reversible mechanoresponsive luminescent switching. Angew Chem Int Ed, 2020, 59: 17580-17586

50 Zhang H, Chen PZ, Niu LY, et al. A difluoroboron $\beta$-diketonate-based luminescent material with tunable solid-state emission and thermally activated delayed fluorescence. Mater Chem Front, 2020, 4: 285-291

51 Zhu JY, Li CX, Chen PZ, et al. A polymorphic fluorescent material with strong solid state emission and multi-stimuli-responsive properties. Mater Chem Front, 2020, 4: 176-181

Acknowledgements This work was supported by the National Key R\&D Program (2017YFA0204503), the National Natural Science Foundation of China (91833306, 51903186, 21875158, 51633006, and 51733004), and China Postdoctoral Science Foundation (2019T120183 and 2021M692381).

Author contributions Sun L, Yang F, Zhang X, and Hu W conceived and supervised this study; Zhang Y, Wu H, Zheng L, Li S, and Liu S wrote the paper; $\mathrm{Wu} \mathrm{H}$ and Zhang Y performed the material preparation and characterization. Sun Y performed theoretical calculations. All authors contributed to discussion and manuscript review. 
Conflict of interest The authors declare that they have no conflict of interest.

Supplementary information Supporting data are available in the online version of the paper.

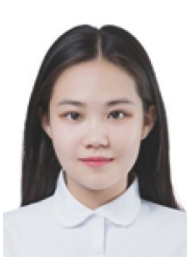

Yihan Zhang is a master's student at Tianjin Key Laboratory of Molecular Optoelectronic Sciences, Department of Chemistry, Tianjin University. Her research interests are organic optoelectronic materials and devices.

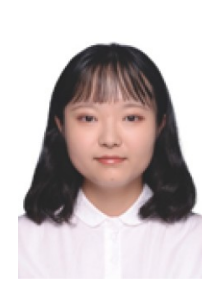

Hongnan $\mathbf{W u}$ is a master's student at Tianjin Key Laboratory of Molecular Optoelectronic Sciences, Department of Chemistry, Tianjin University. From 2018 to now, she has been conducting her master's research at Tianjin University. Her research involves functionalized stimulusresponsive cocrystals.

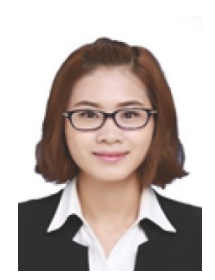

Lingjie Sun is a postdoctoral scholar at the Department of Chemistry, School of Science, Tianjin University. She received her $\mathrm{PhD}$ degree from the same department (supervisor: Prof. Wenping $\mathrm{Hu}$ ). Her interests are in the design, characterization, and optoelectronic properties of organic cocrystals.

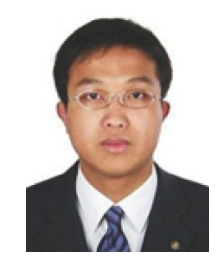

Xiaotao Zhang is a Professor at Tianjin University. He received his $\mathrm{PhD}$ degree from the Institute of Chemistry, Chinese Academy of Sciences, in 2012 after he got his MSc degree (2007) at Zhejiang University. His research interest includes the design and synthesis of novel organic semiconductors, the fabrication, and characterization of organic optoelectronic devices.

\section{共晶工程: 多重刺激下裁剪共晶中电荷转移作用构筑 高灵敏度发光开关材料}

张伊晗 ${ }^{1 \dagger}$, 吴红男 ${ }^{1 \dagger}$, 孙雅静 ${ }^{1}$, 李舒豫 ${ }^{2}$, 刘思 ${ }^{1}$, 郑磊 ${ }^{1}$, 孙玲杰 ${ }^{1,3^{*}}$, 杨方旭 ${ }^{1}$, 张小涛 ${ }^{2,4^{*}}$, 胡文平 ${ }^{1,3}$

摘要 本论文通过溶液自组装方法制备了叭啶- $1,2,4,5$-四氰基苯共晶 (ATC), 这种共晶的发光特性对外部刺激(酸/碱蒸气、研磨和热)表现 出敏感的反应. 通过光谱测试、理论计算和晶体结构研究表明, 交替柱 排列的ATC存在明显的分子间电荷转移相互作用, 这种相互作用在酸 蒸气刺激下减弱, 而在机械研磨外力作用下增强, 实现了外界刺激下分 子间作用力的可逆调控. 此外, 该共晶的响应时间为秒级, 远优于先前 报道的大多数刺激响应型共晶. 因此, ATC 在多种刺激下表现出高灵敏 的苂光切换, 为开发智能材料提供了一种有效的策略. 